\section{Transcranial magnetic stimulation in the treatment of peripartum bipolar depression: a case report}

Rev Bras Psiquiatr. 2018;40:344-345

Brazilian Journal of Psychiatry

CC-BY-NC | doi:10.1590/1516-4446-2018-0037

Treatment of bipolar depression during pregnancy remains a clinical challenge due to the teratogenic risks of mood stabilizers and, to a lesser extent, the risks associated with antidepressants. ${ }^{1}$ However, if left untreated, there is a significantly higher chance of maternal disease relapse and morbidity. ${ }^{2}$ Repetitive transcranial magnetic stimulation (rTMS), currently approved in the United States for treatment of unipolar depression, has shown initial efficacy for both bipolar depression and peripartum unipolar depression. ${ }^{3,4}$ However, there is no known precedent of its use for the treatment of peripartum bipolar depression. We report the case of a patient with bipolar II disorder who received $\mathrm{rTMS}$ for depression during the second and third trimesters of pregnancy.

A 32-year-old G2P1 woman with a history of bipolar II disorder presented at 20 weeks gestation due to peripartum recurrence of depressive symptoms. The patient had a history of predominantly depressive episodes starting from her early 20s, as well as two past suicide attempts via pill overdose and one psychiatric hospitalization for depression with suicidal ideations. She also experienced hypomanic episodes later on, characterized by both irritable and expansive moods, with increased energy, decreased need for sleep, distractibility, as well as shopping and spending sprees. These periods lasted a maximum of 5 days, and never resulted in severe functional impairment nor hospitalization. The patient had no history of manic episodes, substance use, personality disorders, any other psychiatric disorders, or medical comorbidities.
She had a family history of unipolar depression, but no known history of suicide, bipolar disorder, or other psychiatric illnesses.

The patient was intolerant to multiple antidepressants, including paroxetine, venlafaxine, and bupropion; a trial of escitalopram had been unsuccessful. These antidepressant trials had never resulted in mixed depressive symptomatology or switch into hypomania. Notably, she had never received electroconvulsive therapy (ECT), due to self-preference. The patient had previously undergone three successful adjunctive courses of rTMS, with the last course completed a year and a half before presentation. She was on a regimen of lamotrigine $400 \mathrm{mg} /$ day and aripiprazole $15 \mathrm{mg} /$ day for the past year, which was continued throughout her treatment. She had recently completed an intensive outpatient program for depression, but did not continue any psychotherapy during her treatment.

On initial evaluation, the patient reported symptoms of moderately depressed mood, decreased appetite with weight loss, anergia, psychomotor retardation, and occasional passive suicidal ideations without intent or plan. With the exception of mildly slowed psychomotor activity and a dysthymic affect, her mental status exam was unremarkable. There was no evidence of psychosis or a mixed manic episode. Given the patient's previous good response to rTMS and antidepressant intolerance, a course of rTMS was initiated. She received fastfrequency $(10 \mathrm{~Hz})$, 4-second train duration, 16-second intertrain interval, 4000 pulse-per-session (pps) treatments on the left dorsolateral prefrontal cortex (DLPFC), followed by slow-frequency $(1 \mathrm{~Hz}), 300$-second train duration, 60-second intertrain interval, 900-pps treatments on the right DLPFC. Treatments were delivered using a Mag Pro R30 simulator (Tonica Electronik A/S, Farum, Denmark) with a cool-B65 figure-of-eight coil at $120 \%$ of the observed motor threshold.

Altogether, the patient received 30 rTMS treatments over 6 weeks, followed by maintenance treatments every other week until delivery, for a total of 41 sessions. She tolerated rTMS extremely well and did not have any side effects or emergent manic symptoms. The patient reported resolution of suicidal ideations, marked improvement

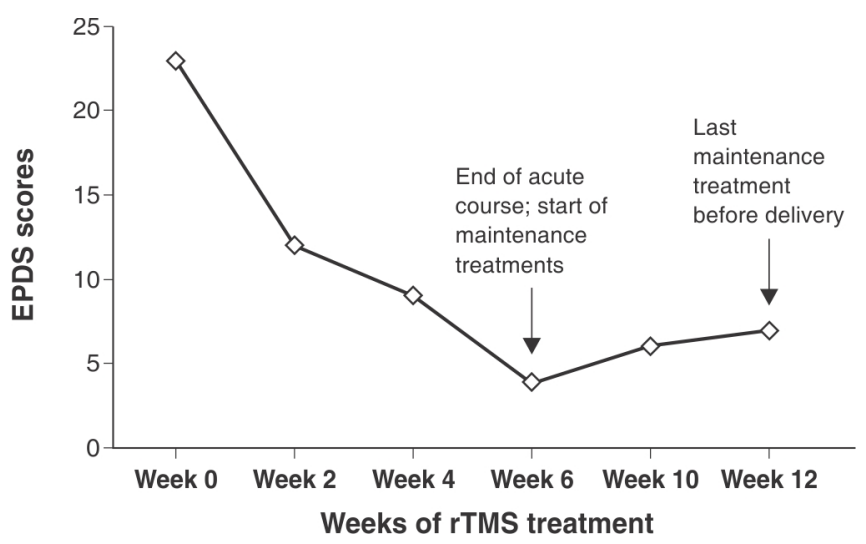

Figure 1 Edinburgh Postnatal Depression Scale (EPDS) scores over time.Scores 0-9 normal, 10-30 possible depression. 
in depressive symptoms (particularly dysphoria and anergia), and improved overall daily functioning. The progressive decline in her Edinburgh Postnatal Depression Scale (EPDS) scores over the course of treatment can be seen in Figure 1. The patient delivered a healthy full-term infant, was symptomatically stable postpartum, and reported sustained improvement of depression on a follow-up phone call 6 weeks post-delivery.

To our knowledge, this is the first documented report of rTMS as a potential treatment option in peripartum bipolar depression. Women with bipolar disorder are at high risk for peripartum relapse and associated morbidity. ${ }^{2}$ Effective treatment is critical, but clinicians must consider the risks of pharmacotherapy to both the mother and fetus. This case highlights the usefulness and tolerability of rTMS as a potential treatment for women with moderate peripartum bipolar depression. Equally importantly, it illustrates the importance of working with patients in a collaborative fashion to weigh the risks and benefits of treatment interventions, including maintaining autonomy to decline ECT.

Because rTMS does not involve transplacental passage, it poses a significant advantage over pharmacological treatment. Likewise, rTMS may be advantageous over ECT because it does not involve the use of anesthetics or intubation, which may be associated with maternal and neonatal complications. ${ }^{5}$ Nevertheless, with current evidence, the role of rTMS in patients with severe depression or active suicidality - where ECT would normally serve as first-line treatment - is still unclear. Although this case adds to the documentation of safety and tolerability of rTMS during pregnancy for both mother and baby, extensive research is still needed to fully elucidate the role of rTMS treatment in this unique patient population.
Willa Xiong, ${ }^{1}$ Ruthzaine Lopez, ${ }^{2}$ Pilar Cristancho ${ }^{3}$ ${ }^{1}$ Department of Psychiatry, New York University, New York, NY, USA. ${ }^{2}$ Facultad de Medicina, Universidad Adventista del Plata, Entre Ríos, Argentina. ${ }^{3}$ Department of Psychiatry, Washington University School of Medicine in St. Louis, St. Louis, MO, USA.

Submitted Jan 26 2018, accepted Apr 022018.

\section{Acknowledgements}

Research reported in this publication was supported by the National Institute of Mental Health (grant 3R34MH 101433 to Washington University), The Center for Brain Research in Mood Disorders at Washington University, and The Foundation for Barnes-Jewish Hospital.

\section{Disclosure}

The authors report no conflicts of interest.

\section{References}

1 Chisolm MS, Payne JL. Management of psychotropic drugs during pregnancy. BMJ. 2016;532:h5918.

2 Viguera AC, Cohen LS, Baldessarini RJ, Nonacs R. Managing bipolar disorder during pregnancy: weighing the risks and benefits. Can $\mathrm{J}$ Psychiatry. 2002;47:426-36.

3 McGirr A, Karmani S, Arsappa R, Berlim MT, Thirthalli J, Muralidharan $\mathrm{K}$, et al. Clinical efficacy and safety of repetitive transcranial magnetic stimulation in acute bipolar depression. World Psychiatry. 2016;15:85-6.

4 Kim DR, Epperson N, Paré E, Gonzalez JM, Parry S, Thase ME, et al. An open label pilot study of transcranial magnetic stimulation for pregnant women with major depressive disorder. J Womens Health (Larchmt). 2011;20:255-61.

5 Anderson EL, Reti IM. ECT in pregnancy: a review of the literature from 1941 to 2007. Psychosom Med. 2009;71:235-42. 